\title{
The effectiveness of preemptive analgesia for relieving postoperative pain after video-assisted thoracoscopic surgery (VATS): a prospective, non-randomized controlled trial
}

\author{
Min Kong ${ }^{1,2}$, Xiaodong $\mathrm{Li}^{1}$, Jianfei Shen ${ }^{1}$, Minhua Ye ${ }^{1}$, Haifei Xiang ${ }^{3}$, Dehua $\mathrm{Ma}^{1,2}$ \\ ${ }^{1}$ Department of Thoracic Surgery, Taizhou Hospital of Zhejiang Province affiliated to Wenzhou Medical University, Taizhou, China; ${ }^{2}$ Department of \\ Thoracic Surgery, Enze Hospital, Taizhou Enze Medical Center (Group), Taizhou, China; ${ }^{3}$ Department of Anesthesiology, Enze Hospital, Taizhou \\ Enze Medical Center (Group), Taizhou, China \\ Contributions: (I) Conception and design: M Kong, D Ma; (II) Administrative support: None; (III) Provision of study materials or patients: D Ma, M \\ Ye; (IV) Collection and assembly of data: X Li, H Xiang; (V) Data analysis and interpretation: M Kong, J Shen; (VI) Manuscript writing: All authors; \\ (VII) Final approval of manuscript: All authors. \\ Correspondence to: Dehua Ma. Department of Thoracic Surgery, Taizhou Hospital of Zhejiang Province affiliated to Wenzhou Medical University, \\ Taizhou, China. Email: tzyyxwk@163.com.
}

Background: The effectiveness of preemptive analgesia (PA) for relieving postoperative pain and reducing the side effects of analgesics following video-assisted thoracoscopic surgery (VATS) has not yet been determined. This study intends to test the clinical application value of PA in the perioperative period of VATS.

Methods: From January 2018 to August 2018, we divided patients who underwent VATS in our hospital into a trial group (PA group) and a control group (traditional analgesia group, TA group). The PA group received a PA program, and the TA group was administered a conventional postoperative analgesia scheme. We compared the two groups according to the intensity of postoperative pain using the numeric rating scale (NRS), the incidence rate of analgesic drug-related adverse reactions, and the severity of stress-induced inflammation.

Results: One hundred five cases from the PA group, and 80 cases from the TA group were included in the analysis. There were no significant differences between the two groups in baseline characteristics $(\mathrm{P}>0.05)$. The PA group had a lower incidence rate of side effects from the analgesics compared to the TA group, and there was a statistical difference at 48 and 72 hours after surgery $(\mathrm{P}<0.05)$. The PA group had a slightly lower score than the TA group for postoperative resting pain. However, this difference was not statistically significant $(\mathrm{P}>0.05)$. The motion pain NRS score of the PA group was lower than the TA group, and although there were no significant differences at 4,24 , and 48 hours $(P>0.05)$, there was a statistically significant difference at 72 hours $(\mathrm{P}<0.05)$. In the subset of patients with motion pain NRS $\geq 3$ points, the PA group was marginally higher than the TA group at 4 hours $(\mathrm{P}>0.05)$ but was lower than the TA group at 24, 48, and 72 hours, with a statistically significant difference at 24 and 72 hours $(\mathrm{P}<0.05)$. There were no statistically significant differences in perioperative stress indexes between the two groups $(\mathrm{P}>0.05)$.

Conclusions: PA can relieve postoperative pain following VATS and reduce the incidence rate of analgesic drug-related adverse effects.

Keywords: Preemptive analgesia (PA); thoracoscopy; side effects; clinical research

Submitted Jul 02, 2020. Accepted for publication Aug 26, 2020.

doi: $10.21037 /$ jtd-20-2500

View this article at: http://dx.doi.org/10.21037/jtd-20-2500

(C) Journal of Thoracic Disease. All rights reserved. 


\section{Introduction}

Thoracic tumors, especially lung cancers, are among the most prominent malignancies endangering human health and life, with rapidly increasing morbidity and mortality (1). Video-assisted thoracic surgery (VATS) is a required surgical method to treat lung cancer and other thoracic diseases and offers several potential advantages compared to traditional thoracotomy. VATS can reduce surgical trauma, shorten surgery, and anesthesia time, and decrease the incidence rate of cardiopulmonary complications $(2,3)$. With the progress of VATS technology and the improvement of VATS instruments, VATS is increasingly widely used. However, patients still require analgesia after VATS $(4,5)$. Postoperative pain is still one of the most common clinical problems following VATS and is a key factor hindering the rapid recovery of patients $(6,7)$.

The clinical significance of improving postoperative analgesia is self-evident. However, the multi-source mechanism of postoperative pain following VATS presents a persistent challenge for clinicians. This phenomenon is because the single analgesic method or the calibration of analgesic drugs cannot achieve an ideal clinical result, with postoperative pain (and especially motion pain) being problematic. According to the statistics of the pain management team in our center, $80 \%$ of the 375 patients who underwent VATS from January 2016 to June 2016 had a numeric rating scale (NRS) $\geq 3$ within 3 days after surgery using the current routine postoperative analgesia program.

Preemptive analgesia (PA) refers to using analgesic methods before surgical incision or stimulation to change the perception of harmful stimulation by peripheral and central nervous systems; to reduce central sensitization, hyperalgesia, and touch-induced pain. However, this concept is still controversial (8-11). Some studies suggest using analgesia before surgery to reduce postoperative pain, and opioid use is unnecessary (8). Some studies have also confirmed the effectiveness of preemptive analgesia in VATS, but the numbers of cases and diseases involved are relatively small (12). The effectiveness of PA in relieving postoperative pain following VATS and reducing the side effects of analgesics (especially opioid-related side effects) has not yet been determined. Therefore, this study aims to test the clinical application value of PA in relieving postoperative pain following VATS through a prospective, non-randomized controlled clinical study.

We present the following article following the TREND reporting checklist (available at http://dx.doi.org/10.21037/ jtd-20-2500).

\section{Methods}

\section{Inclusion criteria}

From January 1, 2018, to August 31, 2018, patients who underwent VATS at Enze Hospital of Taizhou Enze Medical Center (Group) were included in our study. Screening criteria included: (I) American Society of Anesthesiologists (ASA) grade I-III; (II) age between 18-80 years; (III) patients with lung or mediastinal disease undergoing VATS; (IV) an expected surgery time of between 1-6 hours; (V) numbers of holes in thoracoscopy is $1-3$.

Exclusion criteria included: (I) patients with chronic obstructive pulmonary disease (COPD), type 2 diabetes mellitus, or chronic pain before surgery; (II) patients taking immunosuppressive drugs before surgery; (III) patients with mental disorders or other causes of non-cooperation; (IIV) participants in clinical studies of other drugs in the past three months; (V) hemoglobin ( $\mathrm{Hb})<80 \mathrm{~g} / \mathrm{L}$.

Rejection criteria: (I) patients who did not meet the inclusion criteria, or met the exclusion criteria after inclusion; (II) patients who were converted to thoracotomy, or had massive bleeding and required a blood transfusion of over $400 \mathrm{~mL}$; (III) patients who had severe adverse reactions or accidents during anesthesia or surgery; (IV) patients who needed to be transferred to the intensive care unit (ICU) for transitional treatment following surgery; $(\mathrm{V})$ patients whose clinical information was incomplete.

This study is a single-center, prospective, open, nonrandomized controlled clinical trial. The medical ethics committee of Enze Hospital of Taizhou Enze Medical Center (Group) reviewed and approved the study and registered in the ClinicalTrials.gov Registration (Registration No. chictr2000030144), with the informed consent of all participants.

All procedures performed in this study involving human participants were in accordance with the Declaration of Helsinki (as revised in 2013).

\section{Trial group-PA group}

\section{PA scheme}

The following analgesic treatments were administered either individually or in combination:

(I) Celecoxib $200 \mathrm{mg}$ was taken (orally) in the evening before surgery and on the morning of surgery.

(II) Before surgery, a paravertebral nerve block was performed by injecting $0.375 \%$ ropivacaine $15-30 \mathrm{~mL}$ into $\mathrm{T} 7 / \mathrm{T} 5$ under the guidance of 
B-ultrasound in the anesthesia preparation room.

(III) Before surgery, local infiltration anesthesia was used for PA; $2 \mathrm{~mL} 0.75 \%$ ropivacaine was injected subcutaneously at each incision site.

\section{Postoperative analgesia}

(I) Analgesic method and formula: a routine indwelling precise intravenous analgesia pump was used. The capacity of the pump was $300 \mathrm{~mL}$, including $250 \mu \mathrm{g}$ sufentanil, $250 \mathrm{mg}$ flurbiprofen axetil, $4 \mathrm{~mL} / \mathrm{h}$ continuous dosage, and $4 \mathrm{~mL} / \mathrm{h}$ bolus dosage (the longest lasting time was 72 hours).

(II) Postoperative ward analgesia program: for an NRS $\leq 3$, the bolus dosage button was pressed before coughing activity, and the dosage of the analgesia pump was then added. For an NRS 4-6: the bolus dosage button was pressed, and the dosage of the analgesia pump was then added (with reassessment after 5 minutes). If this was ineffective in relieving pain, flurbiprofen axetil $50 \mathrm{mg}$ or morphine $2 \mathrm{mg}$ slow intravenous injection was administered (with reassessment after 5-10 minutes). For NRS $>6$ : the acute pain services (APS) physician and anesthesiologist were contacted for a consultation to aid in adjusting the analgesic program; the above adjustments are recorded.

See appendix (Supplementary file) for details regarding the observation and evaluation of the preventive antiemetic and the treatment plan for adverse effects of analgesia (including the treatment of circulatory inhibition, respiratory inhibition, excessive sedation, and nausea and vomiting). Pain score, sedation score, and adverse drug reactions were tested every 4 hours.

\section{Control group-traditional analgesia (TA) group}

No PA was used for the control. Postoperative analgesia used in this group included: an intravenous analgesia pump and postoperative ward analgesia program, preventive antiemetic, observation and evaluation in the recovery room, the treatment plan for adverse reactions of analgesia, and dynamic evaluation record. It is the same postoperative analgesia program used in the trial group.

\section{Anesthesia and operation methods}

All operations were performed by the same team of surgeons and anesthetized by the same team of anesthesiologists.
(I) Anesthesia method: all patients were supported using inhalation and intravenous anesthesia. Following anesthesia, central vein catheterization was performed to set up a rapid fluid supply pathway. The surgeons monitored arterial blood pressure through radial artery catheterization. During surgery, single contralateral lung ventilation was used, and blood pressure was supported within the normal range by adjusting the concentration of the anesthetic and by using vasoactive drugs. No other anesthetic drugs or methods were used.

(II) Surgery method: one hole, two holes, and three holes can be used in VATS of pulmonary diseases. The onehole design method takes the $5^{\text {th }}$ intercostal space of the axillary front line as the endoscopic and operative hole. The two-hole design method takes the $7^{\text {th }}$ intercostal space of the axillary middle line as the endoscopic hole, and the $4^{\text {th }}$ or $5^{\text {th }}$ intercostal space of the axillary front line as the operative hole. The three-hole design method uses the $7^{\text {th }}$ or $8^{\text {th }}$ intercostal space of the axillary posterior line as the endoscopic hole, and the $4^{\text {th }}$ or $5^{\text {th }}$ intercostal space of the axillary front line and the $6^{\text {th }}$ or $7^{\text {th }}$ intercostal space of the subscapular angle line as the operative holes.

In our study, all the anterior mediastinal tumors were resected using the three-hole surgery design. The $5^{\text {th }}$ or $6^{\text {th }}$ intercostal space of the axillary midline was used as the endoscopic hole, and the $5^{\text {th }}$ intercostal space and the $2^{\text {nd }}$ intercostal space between the anterior axillary and the clavicular midline operative holes. The resection of posterior mediastinal tumors was the same as lung tumors. This study did not include endoscopic esophageal surgeries.

\section{Observation indicators}

(I) NRS: NRS (numeric rating scale) is a segmented numeric version of the visual analog scale (VAS) in which a patient selects a whole number $(0-10$ integers) that best reflects the intensity of their pain (9). The patients were taken to the postoperative recovery room for observation following surgery, and then to the ward after tracheal extubation. The NRS scores (including rest and motion pain NRS scores) were recorded at 4, 24, 48, and 72 hours after surgery.

(II) The incidence rate of analgesic-related side effects: adverse reactions related to analgesia, including nausea, vomiting, pruritus, lethargy, arrhythmia, and respiratory depression, were recorded any time they 
occurred.

(III) Perioperative stress indicators, including plasma cortisol, blood glucose, C-reactive protein (CRP), and interleukin-6 (IL-6) concentration: venous blood was drawn before surgery, and again at 4 hours, 1 day, and 2 days after surgery, and sent to the laboratory for analysis.

(IV) Additional dosage of morphine and other analgesics: when the NRS score of the patient is $\geq 5$, morphine $(0.1 \mathrm{mg} / \mathrm{kg})$ can be administered intramuscularly. The dosage and cumulative dosage of morphine at 4,24 , and 48 hours after surgery can be calculated. If other analgesic drugs are used, the name and dosage of other drugs shall be recorded.

(V) The primary aspects of this study were the NRS scores and the secondary indicators, including the incidence rate of analgesic-related side effects and perioperative stress indicators.

\section{Statistical analysis}

\section{Sample size calculation}

By using the optimal test, we determined the test level is bilateral $\alpha=0.05, \beta=0.20$, and the sample ratio of the PA group and the TA group is 1:1. According to the data obtained from the pain management group in our center, assuming that NRS $\geq 3$ within 3 days after VATS is the standard, the current ratio is $80 \%$. We assumed that a $20 \%$ reduction in the proportion of the PA group is clinically significant. The Power and Sample Size Program 3.0 statistical software were used for calculation. A sample size of 79 cases was needed for each group, and $10 \%$ of the dropout rate was added gradually. There were 87 cases in each group, and 174 patients were needed in the two groups in total. In the mid-term analysis, considering the increase of the shedding rate, we continued to expand the sample size. So, 23 cases were added into each group, with 46 cases across both groups. Finally, 220 cases were included in the study, with 110 cases in each group.

\section{Grouping method}

Patients were divided into two groups according to hospital admission. Patients admitted between January 1, 2018, and April 30, 2018, were assigned to the conventional analgesia control group (TA group), and patients admitted between May 1, 2018, and August 31, 2018, was assigned to the PA group (PA group).

\section{Statistical methods}

Data were analyzed using SPSS 22.0 (SPSS Inc., Chicago, USA) and Stata 7.0 (Stata Corp, Texas, USA). Descriptive statistics were used to calculate continuous variables, numbers of observations (n), arithmetic mean/median, standard deviation/quartile spacing, and minimum and maximum values of variables. The $t$-test or nonparametric test (Wilcoxon test) was used to compare the two groups. For discontinuous variables (or classified variables), descriptive statistics calculated the number and frequency. A comparison between the two groups was conducted using the Pearson chi-square test or Fisher's exact test. $\mathrm{P}<0.05$ was considered statistically significant.

\section{Results}

\section{Enrolment}

Two hundred twenty patients took part in the trial, with 110 in each group. After exclusion and elimination, 105 cases from the PA group and 80 cases from the TA group were included in the analysis. The TA group had a smaller cohort because blood samples from some patients were not collected, or their clinical information was not gathered completely (Figure 1).

\section{Comparison of baseline characteristics between the two groups}

There were no significant differences between the two groups in age, sex, disease type, operation mode, incision number, general anesthesia duration, surgery duration, number of closed thoracic drainage tubes (CTDT), chest tube retention time, and postoperative hospital stay $(\mathrm{P}>0.05)$ (Table 1).

\section{Comparison of pain NRS between the two groups}

In the subset of patients with postoperative motion pain NRS $\geq 3$, the PA group was slightly higher than the TA group at 4 hours post-surgery $(\mathrm{P}>0.05)$. However, the PA group was lower than the TA group at 24,48 , and 72 hours post-surgery, and the difference was statistically significant at 24 and 72 hours $(\mathrm{P}<0.05)$ (Figure 2).

For the NRS of postoperative resting pain, the PA group was marginally lower than the TA group at 4, 24, 48, and 72 hours, though the differences were not statistically significant $(\mathrm{P}>0.05)$. The NRS of postoperative motion 


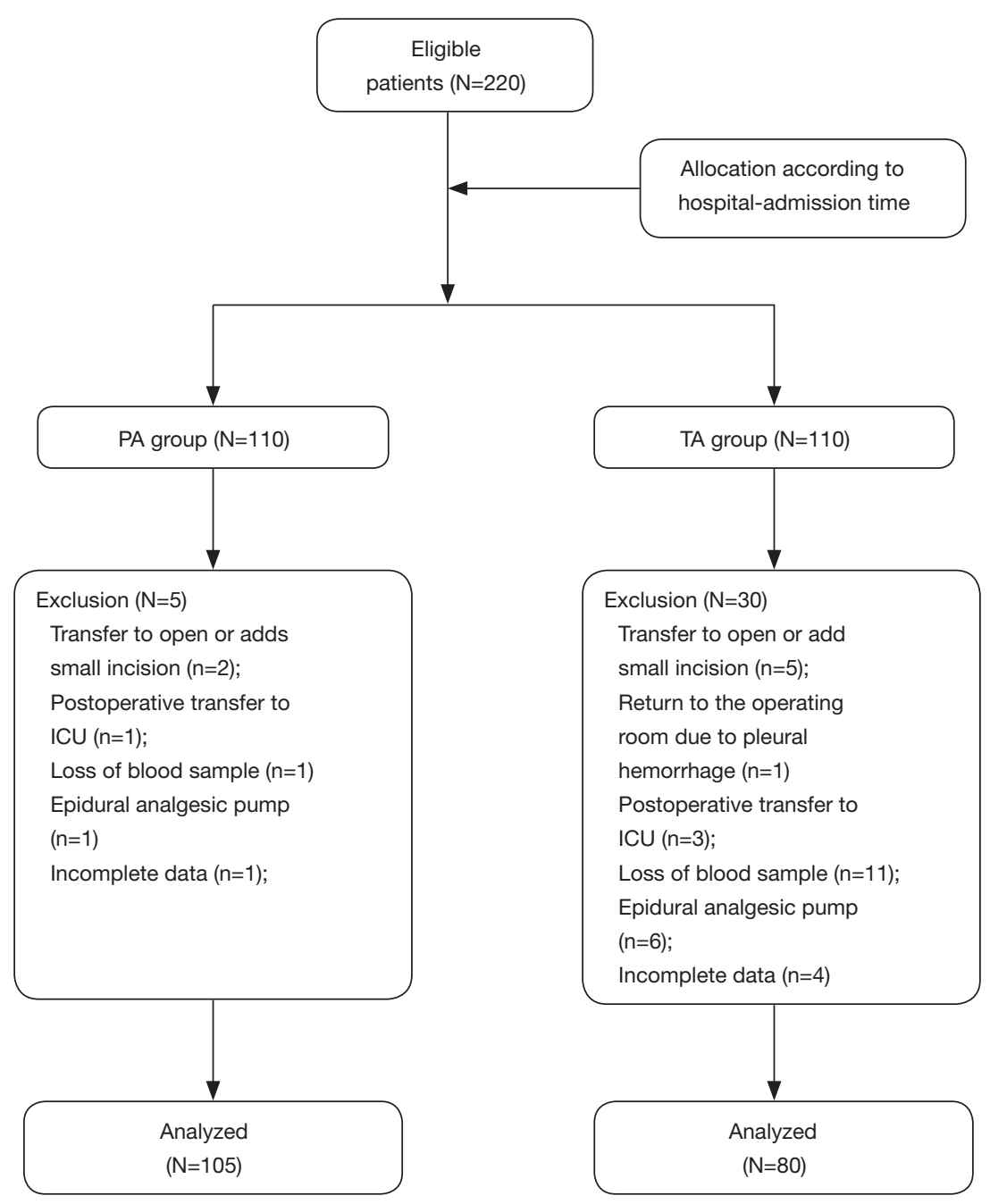

Figure 1 Trial profile. PA, preemptive analgesia; TA, traditional analgesia.

pain in the PA group was lower compared to the TA group. The differences at 4,24 , and 48 hours were not statistically significant $(\mathrm{P}>0.05)$. However, the difference at 72 hours was statistically significant $(\mathrm{P}<0.05)$ (Figure 3).

\section{Comparison of pain site and analgesic-related side effects between the two groups}

For both groups, the most common site of postoperative pain was at the incision site. There were no statistically significant differences between the groups at 4, 24, 48, and 72 hours following surgery $(\mathrm{P}>0.05)$.

Nausea, vomiting, and dizziness were the common side effects of analgesics in both groups. The incidence rate of analgesic-related side effects in the PA group was lower than the TA group, and there was a statistically significant difference at 48 and 72 hours post-surgery $(\mathrm{P}<0.05)$ (Table 2).

\section{Comparison of stress indicators between the two groups}

There were no statistically significant differences in the perioperative stress indexes, including blood glucose, plasma cortisol, CRP, and IL-6 between the two groups ( $\mathrm{P}>0.05)$ (Table 3).

\section{Discussion}

In this study, we found that PA can relieve the postoperative pain of VATS and reduce the incidence rate of analgesicrelated adverse reactions compared with the traditional 
Table 1 Baseline demographic and clinical characteristics of two groups

\begin{tabular}{|c|c|c|c|}
\hline Characteristic & PA group ( $N=105)$ & TA group $(\mathrm{N}=80)$ & $\mathrm{P}$ \\
\hline Gender (M/F) & $46 / 59$ & $33 / 47$ & 0.73 \\
\hline Weight (kg) & $62 \pm 12.28$ & $62 \pm 9.50$ & 0.92 \\
\hline Disease type, no. (\%) & & & 0.35 \\
\hline Pulmonary benign nodules & $19(18 \%)$ & $10(13 \%)$ & \\
\hline Pulmonary bulla & $12(11 \%)$ & $7(9 \%)$ & \\
\hline Mediastinal tumor & $6(6 \%)$ & $11(14 \%)$ & \\
\hline Major surgical methods, no. (\%) & & & 0.75 \\
\hline Pulmonary bulla resection & $11(10 \%)$ & $10(13 \%)$ & \\
\hline Mediastinal tumor resection & $7(7 \%)$ & $9(11 \%)$ & \\
\hline Pleural dissection/biopsy & $7(7 \%)$ & $4(5 \%)$ & \\
\hline The number of incisions, no. (\%) & & & 0.42 \\
\hline Single-ports & $4(4 \%)$ & $1(1 \%)$ & \\
\hline Double-ports & $57(54 \%)$ & $40(50 \%)$ & \\
\hline Three-ports & $44(42 \%)$ & $39(49 \%)$ & \\
\hline Duration of GA (min) & $160(75-320)$ & $153(65-360)$ & 0.36 \\
\hline Retention time of CTDT (d) & $6.9(2-25)$ & $6.5(1-22)$ & 0.11 \\
\hline Postoperative length of stay (d) & $7.9(3-26)$ & $7.5(2-23)$ & 0.11 \\
\hline
\end{tabular}

PA, preemptive analgesia; TA, traditional analgesia; GA, general anesthesia; CTDT, closed thoracic drainage tubes.

analgesia program. However, it has no significant effect on the postoperative stress response.

The goal of perioperative pain management in clinical practice is to relieve the pain experienced by patients and reduce the length of hospitalization to enable rapid recovery following surgery. Severe pain after VATS has a considerable impact on the body and patient habits, including limitation of thoracic activity, shallow breathing, fear of coughing and expectoration, deep breathing, and turning over. It will also be likely to lead to pulmonary complications, including atelectasis and pulmonary infection. Simultaneously, severe pain can also lead to a stress reaction and an increased secretion of catecholamine and other substances, inducing an increase in pulse, respiration, blood pressure, blood sugar, and oxygen consumption. These circumstances will disturb the body's internal stability and weaken immunity, thus delaying recovery. Therefore, effective perioperative pain 


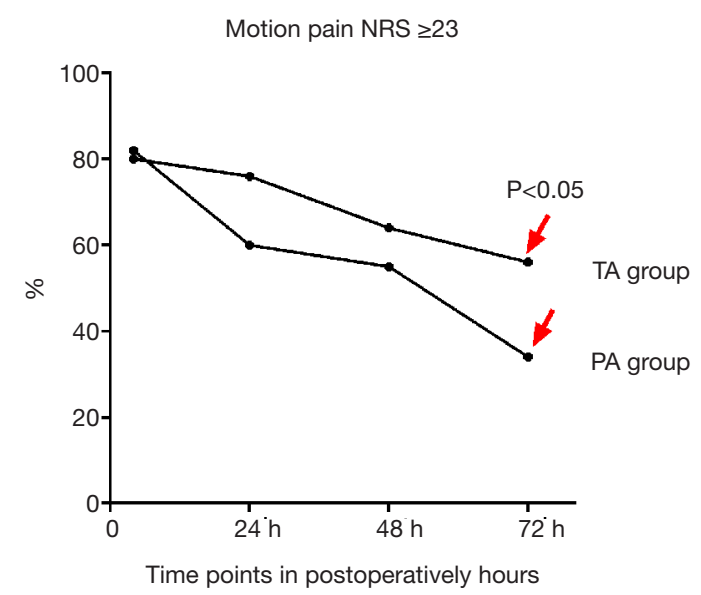

Figure 2 Comparison of the percentage of patients with motion pain NRS $\geq 3$ between the PA and TA groups. NRS, numeric rating scale; PA, preemptive analgesia; TA, traditional analgesia.

management is critical for patients following VATS.

Like other surgeries, thoracoscopic surgery involves many receptors, including skin, muscle, intercostal nerve, rib, and pleura. Because of the direct tissue trauma (i.e., incision, separation, burning, etc.), pain can result from inflammation or direct nerve injury (10). Also, tissue trauma will induce local inflammatory mediators, which will increase the sensitivity of the surrounding area to stimulation (hyperalgesia), or erroneously produce pain to non-noxious stimulation (touch-induced pain). Some inflammatory mediators will also cross the bloodbrain barrier, resulting in the up-regulation of COX2 expression in the spinal cord and cerebral cortex, inducing inflammatory pain $(10,11)$. Specifically, for VATS, the placing of thick and hard materials for thoracic drainage can easily result in stimulation of the pleura and compression of the intercostal nerve, resulting in additional postoperative pain $(13,14)$. Patients need to cough and expectorate actively to prevent atelectasis postsurgery, and so they cannot guarantee protective posture and many other factors, which will also inevitably lead to pain after VATS.

$\mathrm{PA}$ aims to reduce the pain sensitization caused by the stimulation of surgery. The effectiveness of PA is measured by its ability to ease postoperative pain or reduce using analgesics (15). At present, several effective preventive analgesic techniques use various drugs and interventions. These techniques reduce nociceptor activation and inhibit the production and activity of pain neurotransmitters. The result is a reduction in opioid use and opioid-related side effects after surgery (16).

For example, physicians can inject local anesthetics around the surgical incision to offer a preventive analgesic effect. According to a 2005 meta-analysis, patients who received local infiltration anesthesia before incision had a statistically significant decrease in using analgesics and a longer time interval to the first need for remedial analgesia. However, there was no difference in the postoperative pain score (17). Some randomized controlled clinical trials showed that local anesthetics injected around small incisions could reduce postoperative body pain. However, they do not appear to relieve visceral pain (18-21). Preoperative prophylactic administration of anti-inflammatory drugs or analgesics, including non-steroidal anti-inflammatory drugs (NSAIDs)-either selective COX-2 inhibitors or nonselective COX inhibitors-will help reduce the incidence rate of postoperative inflammatory pain. It may also reduce the incidence rate of long-term chronic pain, which might explain the effectiveness of PA in relieving pain following VATS.

As mentioned above, taking celecoxib orally before surgery and using local infiltration anesthesia before incision may reduce pain after VATS (especially motion pain), with benefits being most apparent 72 hours after surgery. The mechanism of preemptive analgesic techniques may be through preventing the transmission of peripheral injury impulses to the central nervous system and inhibiting the establishment of conduction and reducing the peripheral and central sensitization caused by harmful stimulation afferent. Also, PA can reduce the amounts of anesthetics used during surgery. It can also help minimize incrementally increasing doses of the single analgesic method, reducing the incidence rate of analgesicrelated side effects after VATS, especially in patients 48 and 72 hours after surgery.

However, compared with TA, PA does not notably affect the body's stress response following thoracoscopic surgery. These findings show that surgical trauma is still the principal factor causing postoperative stress. It also suggests researchers should explore further measures so that the specifics of PA are perfected to minimize body stress after VATS.

Some limitations of this study should be noted. Because perioperative pain is rooted in patients' subjective feelings, surgical trauma thus not triggers it but is also directly affected by several other factors (including patients' physical conditions and psychological problems). Since this study is 

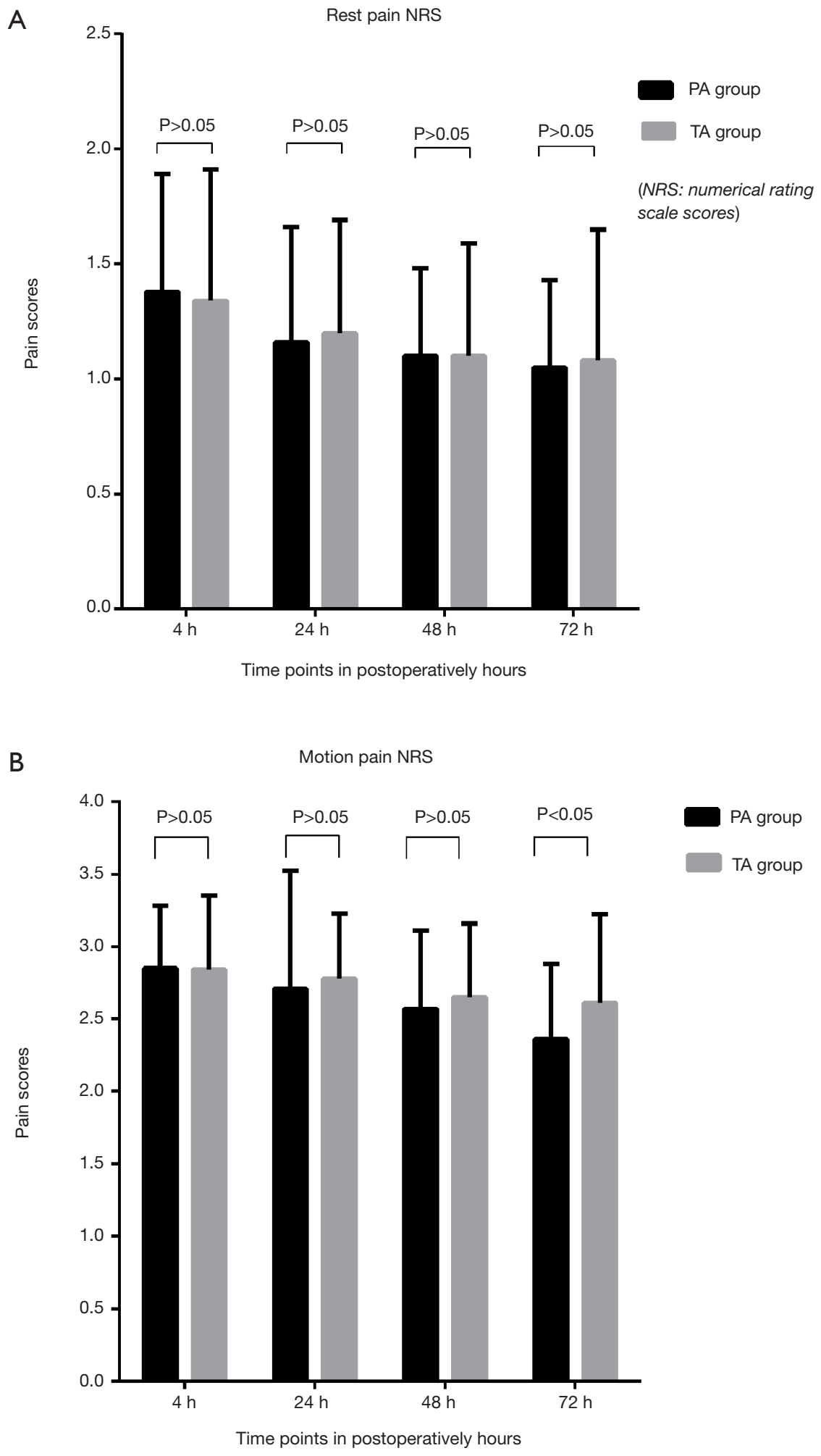

Figure 3 Comparison of pain NRS between PA and TA groups. NRS, numeric rating scale; PA, preemptive analgesia; TA, traditional analgesia. 
Table 2 Pain location and narcotic-related side effects

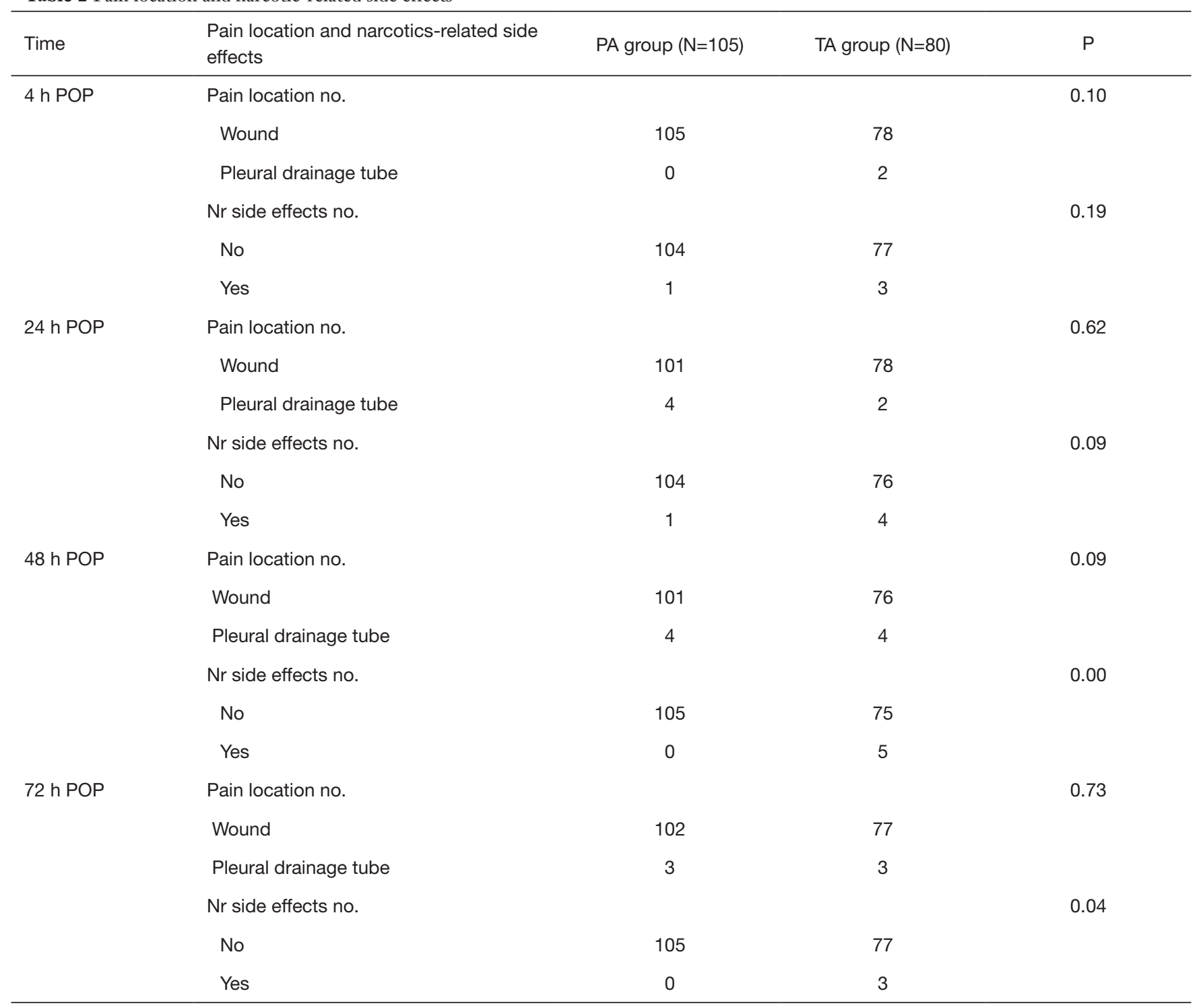

PA, preemptive analgesia; TA, traditional analgesia; POP, postoperatively; Nr, narcotic-related.

not a randomized controlled trial and is limited by sample size, the bias above cannot be excluded entirely.

In conclusion, PA can relieve postoperative pain following VATS, reduce analgesic drug-related side effects, and increase the comfort of patients, promoting rapid postoperative recovery. PA presents a promising treatment mode from now on. The specifics of PA need to be perfected and improved, so it is more conducive to the perioperative pain management of VATS.

\section{Acknowledgments}

Funding: This work was supported by the Taizhou Science and Technology Agency, Zhejiang Province, China (1701KY25).

\section{Footnote}

Reporting Checklist: The authors have completed the 
Table 3 Indicators of physical stress

\begin{tabular}{|c|c|c|c|}
\hline Variables & PA group ( $N=105)$ & TA group $(\mathrm{N}=80)$ & $\mathrm{P}$ \\
\hline Before operation & $5.64 \pm 1.44$ & $5.58 \pm 1.89$ & 0.79 \\
\hline 1st day POP & $7.29 \pm 2.27$ & $7.24 \pm 2.33$ & 0.87 \\
\hline 2nd day POP & $5.97 \pm 1.84$ & $6.00 \pm 1.93$ & 0.89 \\
\hline Before operation & $12.17 \pm 4.21$ & $12.48 \pm 3.30$ & 0.56 \\
\hline 1st day POP & $4.20 \pm 6.42$ & $3.39 \pm 4.37$ & 0.31 \\
\hline 2nd day POP & $6.75 \pm 7.04$ & $6.51 \pm 6.46$ & 0.81 \\
\hline CRP (mg/L) & & & $>0.05$ \\
\hline 2nd day POP & $45.99 \pm 32.35$ & $41.76 \pm 27.79$ & 0.35 \\
\hline IL-6 (pg/mL) & & & $>0.05$ \\
\hline Before operation & $6.68 \pm 15.26$ & $5.76 \pm 10.43$ & 0.64 \\
\hline 1st day POP & $33.21 \pm 72.28$ & $21.26 \pm 16.62$ & 0.56 \\
\hline 2nd day POP & $98.04 \pm 445.38$ & $51.32 \pm 51.34$ & 0.35 \\
\hline
\end{tabular}

PA, preemptive analgesia; TA, traditional analgesia; POP, postoperatively; BG, blood glucose; PCL, plasma cortisol levels; CRP, C-reactive protein; IL-6, interleukin-6.

TREND reporting checklist. Available at http://dx.doi. org/10.21037/jtd-20-2500

Data Sharing Statement: Available at http://dx.doi. org/10.21037/jtd-20-2500

Conflicts of Interest: All authors have completed the ICMJE uniform disclosure form (available at http://dx.doi. org/10.21037/jtd-20-2500). The authors have no conflicts of interest to declare.

Ethical Statement: The authors are accountable for all aspects of the work in ensuring that questions related to the accuracy or integrity of any part of the work are appropriately investigated and resolved. All procedures performed in this study involving human participants were following the Declaration of Helsinki (as revised in 2013). The medical ethics committee approved this study of Enze Hospital of Taizhou Enze Medical Center (Group) (No. 20170822), with the informed consent of all participants.

Open Access Statement: This is an Open Access article distributed in accordance with the Creative Commons Attribution-NonCommercial-NoDerivs 4.0 International License (CC BY-NC-ND 4.0), which permits the noncommercial replication and distribution of the article with the strict proviso that no changes or edits are made and the original work is properly cited (including links to both the formal publication through the relevant DOI and the license). See: https://creativecommons.org/licenses/by-nc-nd/4.0/.

\section{References}

1. Siegel RL, Miller KD, Jemal A. Cancer statistics, 2020. CA Cancer J Clin 2020;70:7-30.

2. Bertolaccini L, Rocco G, Crisci R, et al. Enhanced recovery after surgery protocols in video-assisted thoracic surgery lobectomies: the best is yet still to come? J Thorac Dis 2018;10:S493-6.

3. Bédat B, Abdelnour-Berchtold E, Krueger T, et al. Impact of complex segmentectomies by video-assisted thoracic surgery on peri-operative outcomes. J Thorac Dis 2019;11:4109-18.

4. Umari M, Carpanese V, Moro V, et al. Postoperative 
analgesia after pulmonary resection with a focus on videoassisted thoracoscopic surgery. Eur J Cardiothorac Surg 2018;53:932-8.

5. Umari M, Falini S, Segat M, et al. Anesthesia and fast-track in video-assisted thoracic surgery (VATS): from evidence to practice. J Thorac Dis 2018;10:S542-54.

6. Hopkins KG, Hoffman LA, Dabbs AV, et al.

Postthoracotomy Pain Syndrome Following Surgery for Lung Cancer: Symptoms and Impact on Quality of Life. J Adv Pract Oncol 2015;6:121-32.

7. Nobel TB, Adusumilli PS, Molena D. Opioid use and abuse following video-assisted thoracic surgery (VATS) or thoracotomy lung cancer surgery. Transl Lung Cancer Res 2019;8:S373-7.

8. Kelly DJ, Ahmad M, Brull SJ. Preemptive analgesia I: physiological pathways and pharmacological modalities. Can J Anaesth 2001;48:1000-10.

9. Hawker GA, Mian S, Kendzerska T, French M. Measures of adult pain: Visual Analog Scale for Pain (VAS Pain), Numeric Rating Scale for Pain (NRS Pain), McGill Pain Questionnaire (MPQ), Short-Form McGill Pain Questionnaire (SF-MPQ), Chronic Pain Grade Scale (CPGS), Short Form-36 Bodily Pain Scale (SF-36 BPS), and Measure of Intermittent and Constant Osteoarthritis Pain (ICOAP). Arthritis Care Res (Hoboken) 2011;63 Suppl 11:S240-52.

10. Woolf CJ, Chong MS. Preemptive analgesia--treating postoperative pain by preventing the establishment of central sensitization. Anesth Analg 1993;77:362-79.

11. Møiniche S, Kehlet H, Dahl JB. A qualitative and quantitative systematic review of preemptive analgesia for postoperative pain relief: the role of timing of analgesia. Anesthesiology 2002;96:725-41.

12. Fiorelli A, Vicidomini G, Laperuta P, et al. Pre-emptive local analgesia in video-assisted thoracic surgery

Cite this article as: Kong M, Li X, Shen J, Ye M, Xiang H, Ma D. The effectiveness of preemptive analgesia for relieving postoperative pain after video-assisted thoracoscopic surgery (VATS): a prospective, non-randomized controlled trial. J Thorac Dis 2020;12(9):4930-4940. doi: 10.21037/jtd-20-2500 sympathectomy. Eur J Cardiothorac Surg 2010;37:588-93.

13. Bottiger BA, Esper SA, Stafford-Smith M. Pain management strategies for thoracotomy and thoracic pain syndromes. Semin Cardiothorac Vasc Anesth 2014;18:45-56.

14. Chin K, Adhikary S, Forero M. Erector Spinae Plane (ESP) Block: a New Paradigm in Regional Anesthesia and Analgesia. Current Anesthesiology Reports 2019;9:271-80.

15. Katz J, Clarke H, Seltzer Z. Review article: Preventive analgesia: quo vadimus? Anesth Analg 2011;113:1242-53.

16. Ciftci B, Ekinci M, Celik EC, et al. Efficacy of an Ultrasound-Guided Erector Spinae Plane Block for Postoperative Analgesia Management After Video-Assisted Thoracic Surgery: A Prospective Randomized Study. J Cardiothorac Vasc Anesth 2020;34:444-9.

17. Ong CK, Lirk P, Seymour RA, et al. The efficacy of preemptive analgesia for acute postoperative pain management: a meta-analysis. Anesth Analg 2005;100:75773 , table of contents.

18. Ke RW, Portera SG, Bagous W, et al. A randomized, double-blinded trial of preemptive analgesia in laparoscopy. Obstet Gynecol 1998;92:972-5.

19. Ghezzi F, Cromi A, Bergamini V, et al. Preemptive port site local anesthesia in gynecologic laparoscopy: a randomized, controlled trial. J Minim Invasive Gynecol 2005;12:210-5.

20. Updike GM, Manolitsas TP, Cohn DE, et al. Pre-emptive analgesia in gynecologic surgical procedures: preoperative wound infiltration with ropivacaine in patients who undergo laparotomy through a midline vertical incision. Am J Obstet Gynecol 2003;188:901-5.

21. Leung CC, Chan YM, Ngai SW, et al. Effect of preincision skin infiltration on post-hysterectomy pain-a double-blind randomized controlled trial. Anaesth Intensive Care 2000;28:510-6. 


\section{The treatment plan for adverse effects of analgesia \\ Circulation inhibition}

Symptoms: hypotension.

Treatment method:

(I) Dynamic observation of blood pressure and heart rate.

(II) Elimination of the problems of volume and heart function.

(III) In one of the following cases, it is recommended to cease use of the analgesia pump immediately: (i) heart rate and blood pressure decrease more than $30 \%$ in a short period ( $<30$ minutes) (based on the blood pressure 30 minutes $\times 3$ times after returning to the ward); (ii) blood pressure $<80 \mathrm{mmHg}$; (iii) the patient is exhibiting symptoms and evidence of cardio-cerebral ischemia; (iv) the analgesic level continues to increase; (v) the sensory level is higher than the T2 level, and there is a motor disturbance (upper limb muscle strength decreases).

(IV) For any inconclusive hypotension, the analgesic pump can be stopped first, and the cause determined. Once the cause of the analgesic pump is excluded, it is recommended to resume the use of the analgesic pump as soon as possible.

\section{Respiratory inhibition}

Symptoms: respiratory rate $\leq 8$ times/min; $\mathrm{SpO}_{2}<90 \%$; shallow respiration (excluding patients and surgical factors).

Treatment method:

(I) Wake the patient up at once and ask them to breathe actively.

(II) At the same time, opioids should be stopped.

(III) Oxygen should be administered at once.

(IV) Establish an artificial airway or mechanical ventilation when necessary.

(V) According to the degree of respiratory inhibition, inject $0.1-0.2 \mathrm{mg}$ of naloxone intravenously until the inhalation frequency is more than 8 times/min, or the $\mathrm{SpO}_{2}$ is more than $90 \%$.

\section{Excessive sedation}

Symptoms: drowsiness under stable respiratory circulation; unable to wake up continuously, often accompanied by respiratory amnesia.

Treatment method:

(I) Length of stay (Los) sedation score $\geq 3$ points, report to the doctor at once, contact the full-time anesthesiologist of the anesthesiology department, and adjust the parameters of the analgesia pump.

(II) Dynamic evaluation of Los score and respiratory every hour (q1h); (Note: Los scoring standard of postoperative sedation: 1 point: restlessness; 2 points: quiet cooperation; 3 points: lethargy, being able to follow instructions; 4 points: in a sleeping state, but being able to wake up; 5 points: slow respiratory response; 6 points: in a deep sleeping state, not being able to wake up. (2-4 points were considered satisfactory sedation; 5-6 points were considered over-sedation).

\section{Nausea and vomiting}

Treatment method:

(I) Intraoperative prophylaxis: refer to the prophylactic antiemetic program for details.

(II) Considering that nausea is related to the analgesic pump, droperidol $1 \mathrm{mg}$ was the first choice, and granisetron $3 \mathrm{mg}$ was the second.

(III) For patients who vomit: the first choice is intravenous injection of metoclopramide $10 \mathrm{mg}$.

(IV) If nausea and vomiting cannot be entirely controlled by drug treatment, psychological nursing, and suggestion should be given so. 